Supporting information

\title{
Photoprotective Capabilities of Light-Harvesting Complex II Trimers in the Green Alga Chlamydomonas reinhardtii
}

\author{
Eunchul Kim ${ }^{\dagger} \star$, Keisuke Kawakami ${ }^{\S}$, Ryoichi Sato ${ }^{\dagger}$, Asako Ishii ${ }^{\dagger}$, Jun Minagawa ${ }^{* \dagger}$ \\ $\dagger$ Division of Environmental Photobiology, National Institute for Basic Biology, Okazaki 444-8585, Japan \\ †Division of Biological Science, Graduate School of Science, Nagoya University, Chikusa, Nagoya 464-8602, \\ Japan \\ §Research Center for Artificial Photosynthesis, Osaka City University, 3-3-138, Osaka, Japan
}

\section{Contents}

Figure S1. The average sizes of LHCII trimers under separated and aggregated states.

Figure S2. The relationship between intensity ratio of the red component to the peak and the red-shift of the peak.

Figure S3. Protein sequence alignment of LHCII proteins in a green alga (C. reinhardtii) and a plant (A. thaliana).

Figure S4. Homology modeling of LHCII proteins in a green alga (C. reinhardtii) and a plant (A. thaliana).

Figure S5. Comparison of LHCBM2 and LHCBM3 structures in a PSII-LHCII supercomplex of C. reinhardtii.

Figure S6. Purification of the four distinct types of LHCII trimers from C. reinhardtii.

Figure S7. Fluorescence decays for different excitation intensities to investigate the annihilation effect.

Table S1. Fluorescence lifetime components of LHCII trimers. 
A

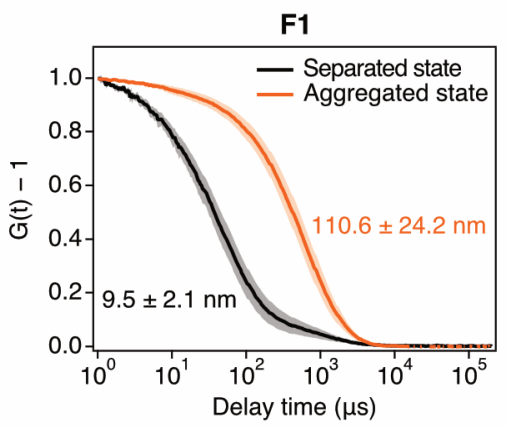

F3

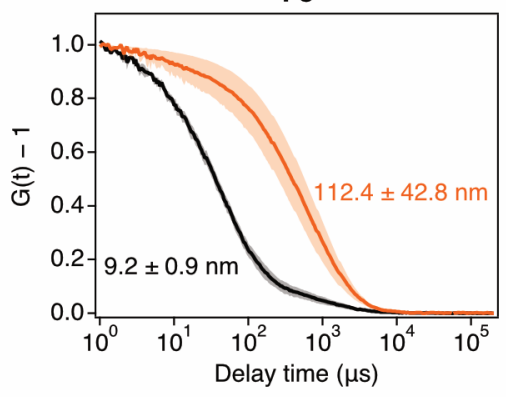

B

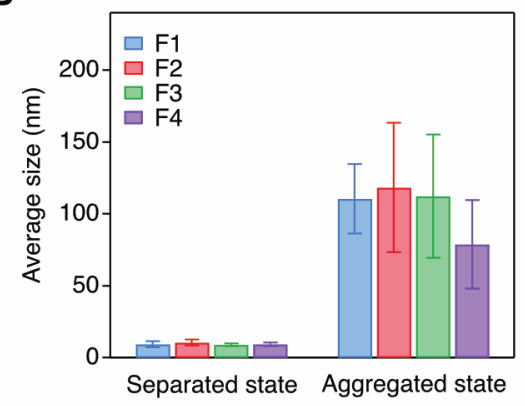

F2

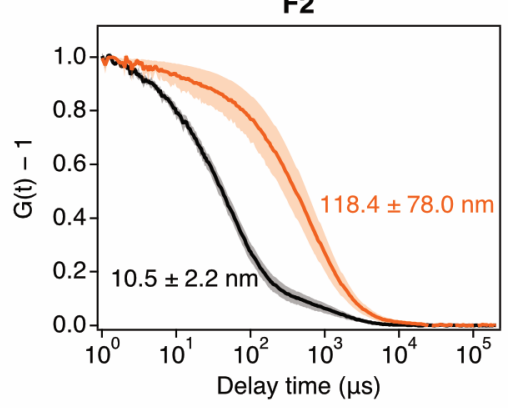

F4

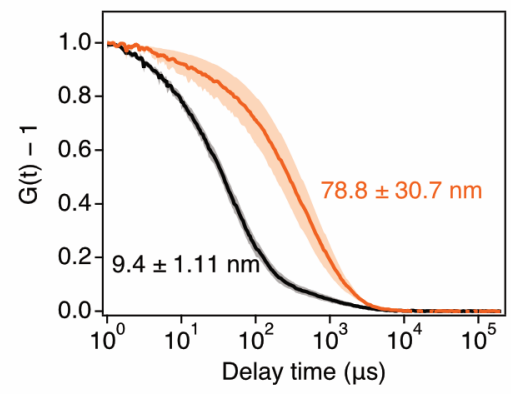

C

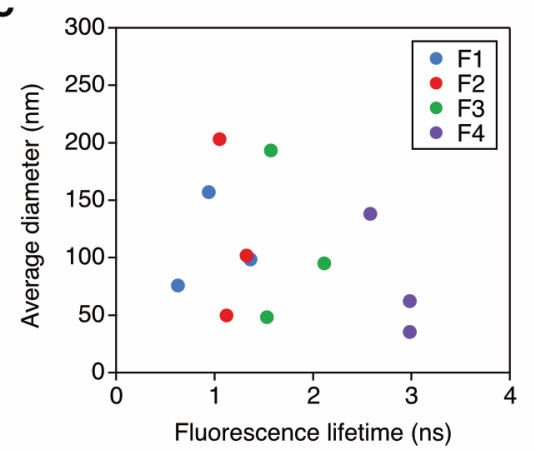

Figure S1. The average sizes of LHCII trimers under separated and aggregated states. (A) Autocorrelation function profiles of F1, F2, F3 and F4 LHCII trimers at $\mathrm{pH} 5.5$ under separated (0.02\% a-DDM) and aggregated states $(0.002 \%$ a-DDM $)$, measured at $20{ }^{\circ} \mathrm{C}$. Shade area represents the standard error range obtained from three biological replicates. Mean \pm STE, obtained from three biological replicates. (B) Average diameter of LHCII trimers under separated and aggregated states. Error range represent standard error obtained from three biological replicates. (C) Relationship between average diameter and fluorescence lifetime of LHCII trimers. 


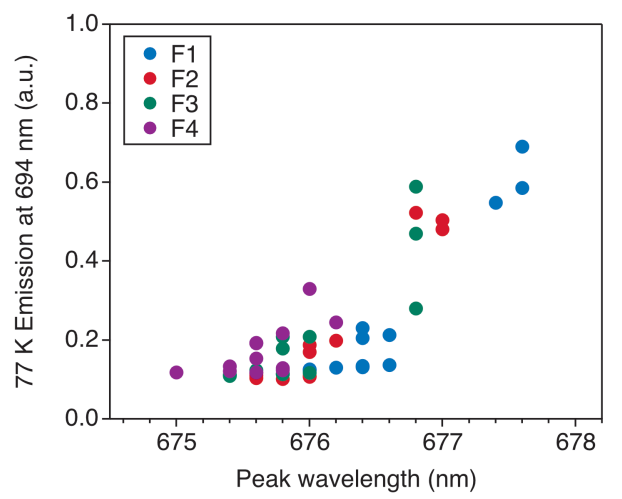

Figure S2. The relationship between intensity ratio of red component to the peak and the red-shift of the peak. Each point obtained from each biological replicate either at $\mathrm{pH} 7.5$ and $\mathrm{pH} 5.5$ under separated and aggregated states. Data were accumulated from three biological replicates. 


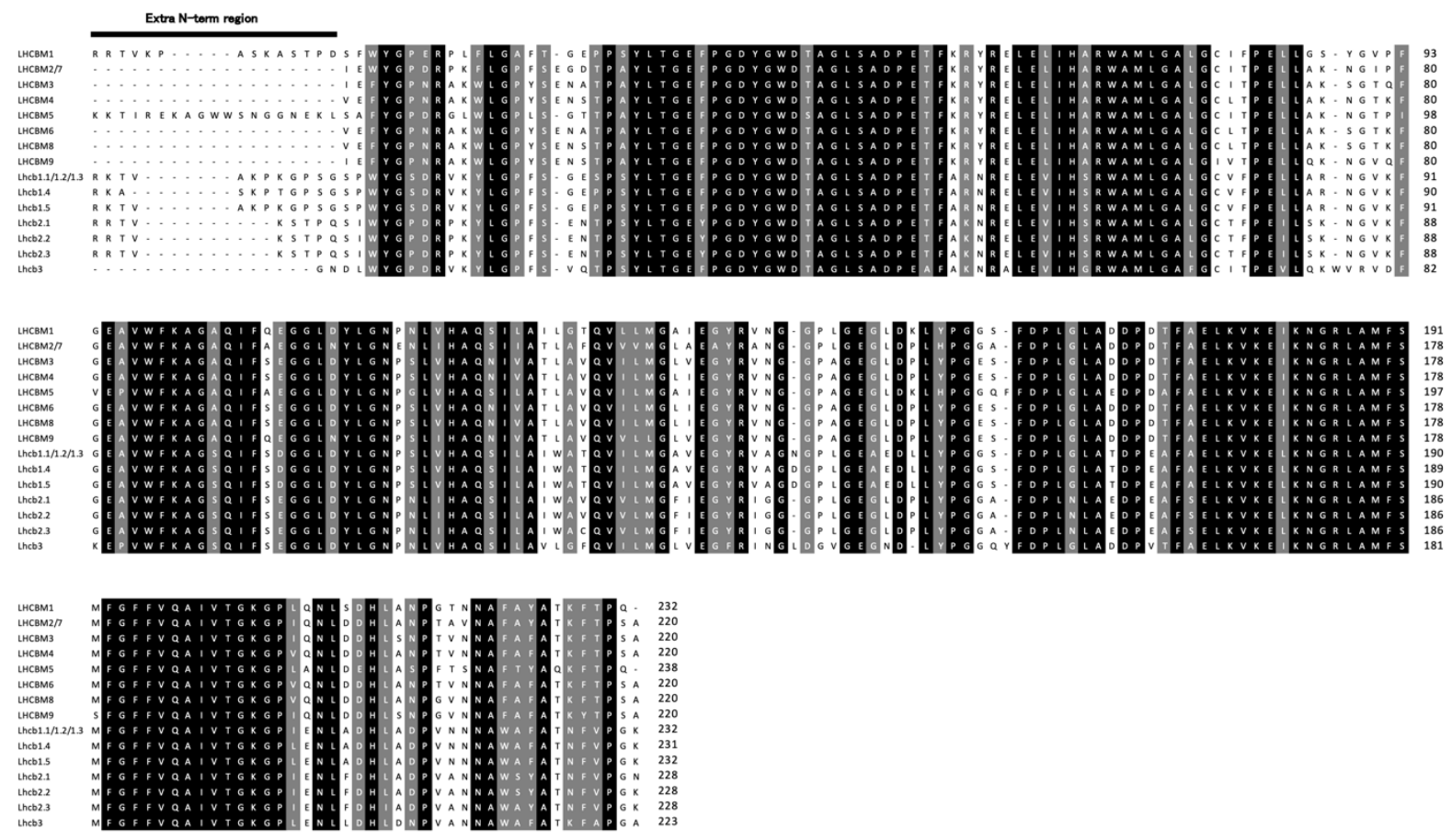

Figure S3. Protein sequence alignment of LHCII proteins in a green alga ( $C$. reinhardtii) and a plant $(A$. thaliana). Mature LHCII protein sequence were referred to Ref. 1 for $C$. reinhardtii LHCs and Ref 2, 3 for $A$. thaliana. The sequences were aligned by MAFFT 7 using G-INS-i option ${ }^{4}$. Black shades indicate highly conserved site in both species. Gray shades indicate conserved site including conservative substitution. Black bar indicates extra $\mathrm{N}$-term regions. 
A
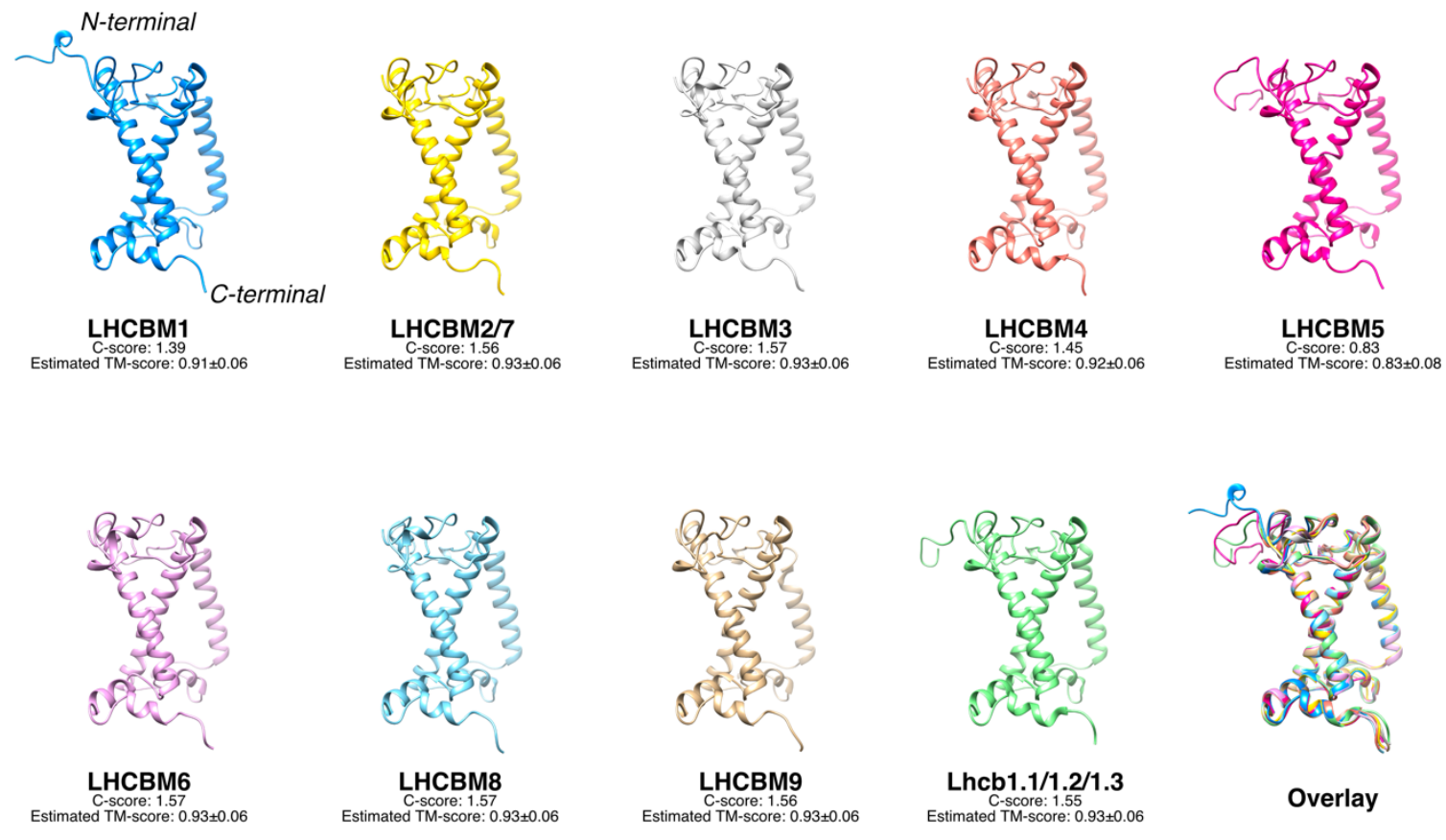

Lhcb1.1/1.2/1.3
Estimated TM-score: $0.93 \pm 0.06$

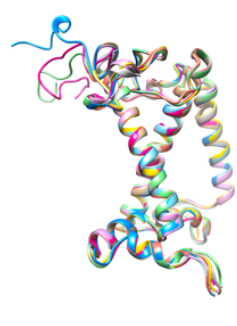

Overlay

B
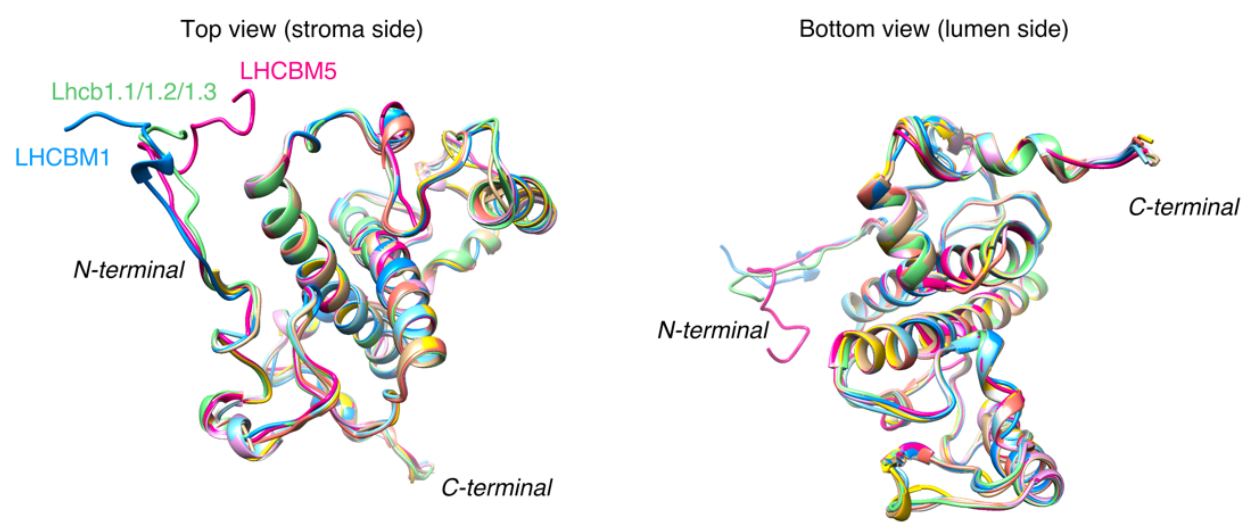

Figure S4. Homology modeling of LHCII proteins in a green alga (C. reinhardtii) and a plant (A. thaliana). (A) The structural models of green algal LHCIIs (LHCBM1-9) and a plant LHCII (Lhcb1.1/1.2/1,3) were generated based on a sequence homology-based structural modeling by using I-TASSER (Ref. 5). Each LHCII model and an overlay of all LHCII models were shown. The protein sequence shown in Fig. S3 were applied to generate the structural models. C-score and estimated TM-score represents the confidence level of the modeling (Ref. 5). (B) Top (stroma side) and bottom (lumen side) views of the overlay. The result of structural modeling clearly shows that LHCBM1, LHCBM5, and Lhcb1.1/1.2/1.3 have extra N-terminal regions whereas C-terminal regions are analogous. 
A LHCBM2 (Type III)

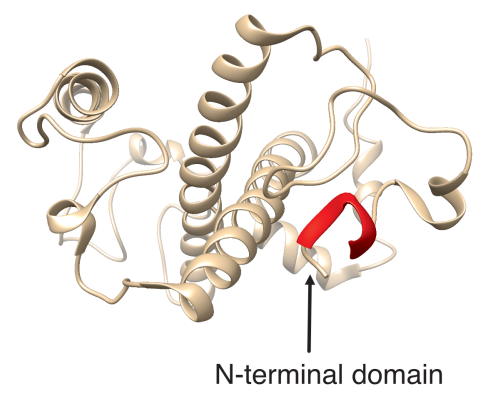

B

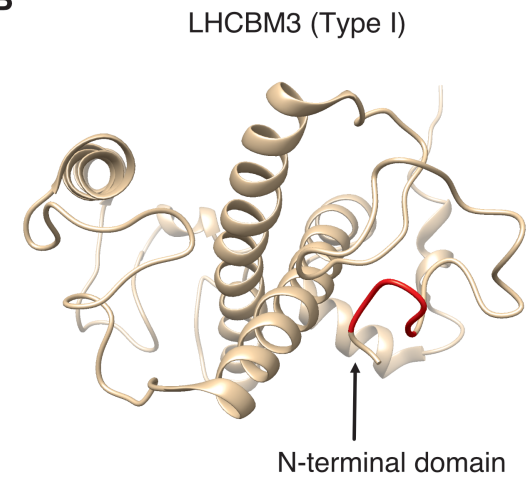

Figure S5. Comparison of LHCBM2 and LHCBM3 structures in a PSII-LHCII supercomplex of $\boldsymbol{C}$. reinhardtii. Structures of LHCBM2 and LHCBM3 were from Ref.6 (PDB:6kac). Images are view from stromal side. Red colored regions indicate the amino acids (Y23-R27) in both LHCBM2 and LHCBM3. 

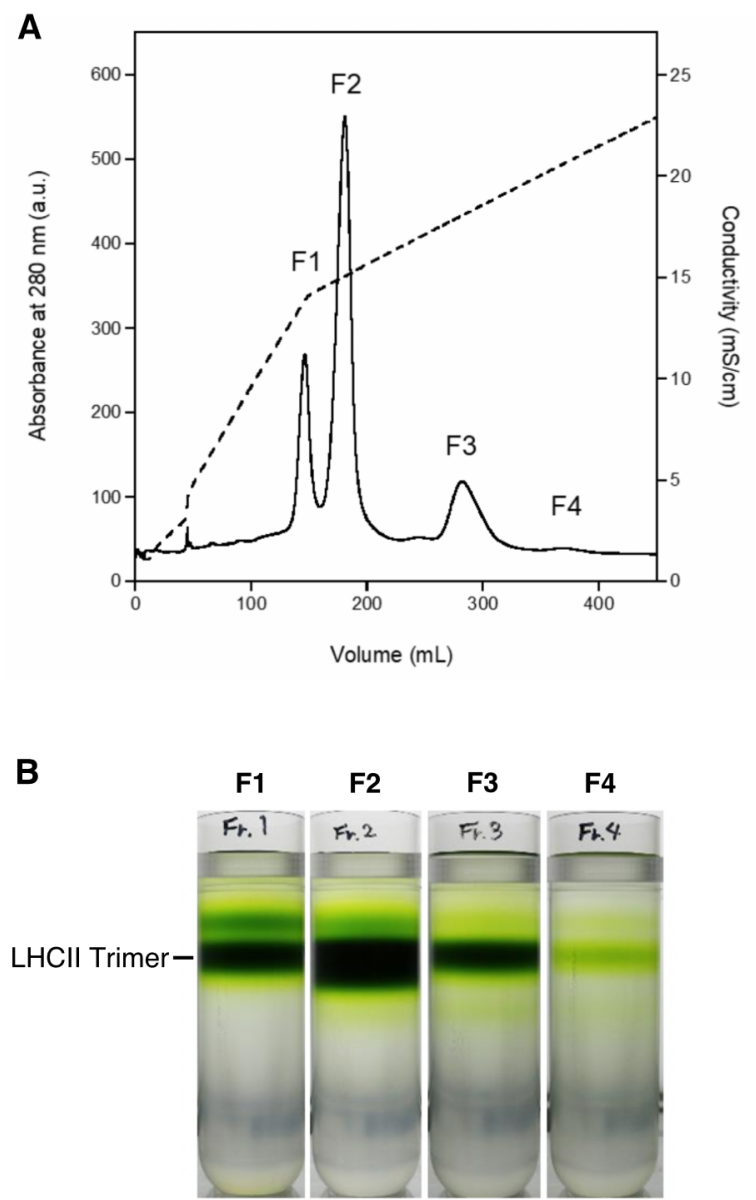

Figure S6. Purification of the four distinct types of LHCII trimers from $\boldsymbol{C}$. reinhardtii. (A) LHCII trimers were separated into four fractions by anion-exchange column chromatography (F1-F4). The solid and dashed lines represent absorption at $280 \mathrm{~nm}$ and conductivity. (B) Each LHCII trimer was isolated by the second sucrose density gradient ultracentrifugation. 

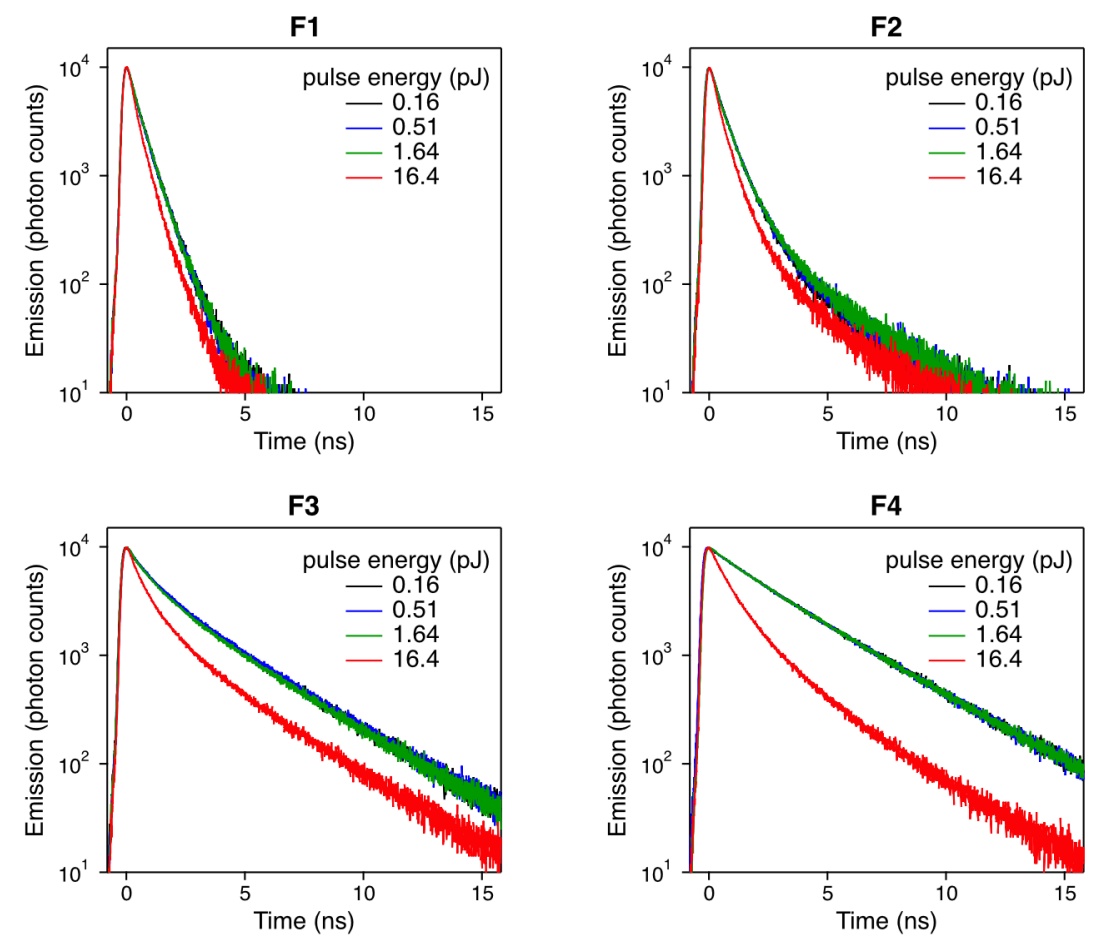

Figure S7. Fluorescence decays for different excitation intensities to investigate the annihilation effect. Fluorescence decays of LHCII trimers under aggregated states at $\mathrm{pH} 5.5$ were obtained by excitation at $463 \mathrm{~nm}$ with varying pulse energies $(0.16,0.51,1.64,16.4 \mathrm{pJ})$. The result represented that there was negligible annihilation effect with the pulse energy of $1.64 \mathrm{pJ}$ while annihilation effect was observed with the pulse energy of $16.4 \mathrm{pJ}$. 
Table S1. Fluorescence lifetime components of LHCII trimers. Fluorescence lifetime and amplitude components were obtained by deconvolution of fluorescence decay with instrument response function (FWHM $80 \mathrm{ps})$. Two and three exponential components were required to obtain best fitting for fluorescence decays obtained under separated and aggregated states, respectively.

\begin{tabular}{|c|c|c|c|c|c|c|c|c|c|c|}
\hline & $\mathrm{pH}$ & $\begin{array}{l}\text { LHCII } \\
\text { trimers }\end{array}$ & $\tau_{1}(\mathrm{~ns})$ & $\mathrm{A}_{1}(\%)$ & $\tau_{2}(\mathrm{~ns})$ & $A_{2}(\%)$ & $\tau_{3}(\mathrm{~ns})$ & $\mathrm{A}_{3}(\%)$ & $\tau_{\text {int.ave }}(\mathrm{ns})$ & $\tau_{\text {amp.ave }}(\mathrm{ns})$ \\
\hline \multirow{8}{*}{ 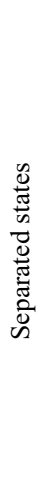 } & \multirow{4}{*}{7.5} & $\mathrm{~F} 1$ & $2.07 \pm 0.03$ & $47 \pm 1$ & $3.86 \pm 0.02$ & $53 \pm 1$ & - & - & $3.29 \pm 0.03$ & $3.03 \pm 0.03$ \\
\hline & & $\mathrm{F} 2$ & $2.04 \pm 0.08$ & $46 \pm 1$ & $3.83 \pm 0.01$ & $54 \pm 1$ & - & - & $3.27 \pm 0.03$ & $3.01 \pm 0.06$ \\
\hline & & F3 & $2.03 \pm 0.03$ & $44 \pm 1$ & $3.84 \pm 0.01$ & $56 \pm 1$ & - & - & $3.30 \pm 0.03$ & $3.03 \pm 0.04$ \\
\hline & & $\mathrm{F} 4$ & $2.06 \pm 0.11$ & $42 \pm 1$ & $3.83 \pm 0.03$ & $58 \pm 1$ & - & - & $3.34 \pm 0.04$ & $3.09 \pm 0.07$ \\
\hline & \multirow{4}{*}{5.5} & $\mathrm{~F} 1$ & $2.63 \pm 0.18$ & $56 \pm 5$ & $4.17 \pm 0.08$ & $44 \pm 5$ & - & - & $3.50 \pm 0.04$ & $3.32 \pm 0.06$ \\
\hline & & $\mathrm{F} 2$ & $2.46 \pm 0.15$ & $48 \pm 2$ & $4.02 \pm 0.03$ & $52 \pm 2$ & - & - & $3.46 \pm 0.04$ & $3.27 \pm 0.07$ \\
\hline & & F3 & $2.57 \pm 0.19$ & $52 \pm 3$ & $4.09 \pm 0.05$ & $48 \pm 3$ & - & - & $3.48 \pm 0.04$ & $3.30 \pm 0.08$ \\
\hline & & $\mathrm{F} 4$ & $2.37 \pm 0.10$ & $44 \pm 2$ & $3.99 \pm 0.03$ & $56 \pm 2$ & - & - & $3.47 \pm 0.04$ & $3.27 \pm 0.07$ \\
\hline \multirow{8}{*}{ 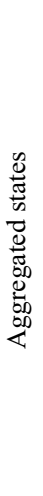 } & \multirow{4}{*}{7.5} & $\mathrm{~F} 1$ & $0.61 \pm 0.02$ & $26 \pm 3$ & $1.88 \pm 0.02$ & $43 \pm 1$ & $3.62 \pm 0.01$ & $31 \pm 3$ & $2.72 \pm 0.07$ & $2.09 \pm 0.09$ \\
\hline & & $\mathrm{F} 2$ & $0.58 \pm 0.01$ & $26 \pm 1$ & $1.84 \pm 0.06$ & $42 \pm 1$ & $3.56 \pm 0.02$ & $32 \pm 1$ & $2.70 \pm 0.02$ & $2.06 \pm 0.03$ \\
\hline & & $\mathrm{F} 3$ & $0.58 \pm 0.02$ & $23 \pm 2$ & $1.96 \pm 0.08$ & $41 \pm 1$ & $3.67 \pm 0.05$ & $36 \pm 2$ & $2.87 \pm 0.01$ & $2.25 \pm 0.01$ \\
\hline & & $\mathrm{F} 4$ & $0.59 \pm 0.06$ & $17 \pm 4$ & $2.10 \pm 0.19$ & $43 \pm 3$ & $3.77 \pm 0.09$ & $40 \pm 3$ & $3.05 \pm 0.07$ & $2.51 \pm 0.15$ \\
\hline & \multirow{4}{*}{5.5} & $\mathrm{~F} 1$ & $0.38 \pm 0.03$ & $68 \pm 4$ & $0.89 \pm 0.06$ & $30 \pm 3$ & $3.55 \pm 0.07$ & $2 \pm 1$ & $0.98 \pm 0.21$ & $0.61 \pm 0.09$ \\
\hline & & $\mathrm{F} 2$ & $0.43 \pm 0.03$ & $65 \pm 1$ & $1.12 \pm 0.06$ & $32 \pm 1$ & $3.37 \pm 0.03$ & $3 \pm 1$ & $1.16 \pm 0.08$ & $0.73 \pm 0.05$ \\
\hline & & F3 & $0.50 \pm 0.09$ & $51 \pm 7$ & $1.54 \pm 0.29$ & $37 \pm 6$ & $3.73 \pm 0.29$ & $12 \pm 2$ & $2.09 \pm 0.29$ & $1.29 \pm 0.30$ \\
\hline & & F4 & $0.42 \pm 0.02$ & $42 \pm 7$ & $1.83 \pm 0.08$ & $28 \pm 3$ & $3.71 \pm 0.03$ & $31 \pm 5$ & $2.85 \pm 0.13$ & $1.82 \pm 0.22$ \\
\hline
\end{tabular}

Means \pm standard error from three biological replicates are shown.

$\mathrm{A}_{\mathrm{i}}$ and $\tau_{\mathrm{i}}$ are amplitude ratio and lifetime of each component, respectively.

$\tau_{\text {int.ave }}$ and $\tau_{\text {amp.ave }}$ are intensity weighted and amplitude weighted average lifetimes, respectively.

$\tau_{\text {int.ave }}=\sum_{i} A_{i} \tau_{i}^{2} / \sum_{i} A_{i} \tau_{i}$

$\tau_{\text {amp.ave }}=\sum_{i} A_{i} \tau_{i} / \sum_{i} A_{i}$ 


\section{Reference}

(1) Elrad, D.; Niyogi, K. K.; Grossman, A. R., A major light-harvesting polypeptide of photosystem II functions in thermal dissipation. Plant Cell 2002, 14, 1801-1816.

(2) Leoni, C.; Pietrzykowska, M.; Kiss, A. Z.; Suorsa, M.; Ceci, L. R.; Aro, E. M.; Jansson, S., Very rapid phosphorylation kinetics suggest a unique role for Lhcb2 during state transitions in Arabidopsis. Plant J. 2013, 76, 236-246.

(3) Jansson, S. A guide to the Lhc genes and their relatives in Arabidopsis. Trends Plant Sci. 1999, 4, $236-240$.

(4) Katoh, Kazutaka, and Daron M. Standley. MAFFT multiple sequence alignment software version 7: improvements in performance and usability. Mol. Biol. Evol. 2013, 30, 772-780.

(5) Yang, J.; Yan, R.; Roy, A.; Xu, D.; Poisson, J.; Zhang, Y.; Song, D., The I-TASSER Suite: Protein structure and function prediction. Nat. Methods 2015, 12, 7-8.

(6) Sheng, X.; Watanabe, A.; Li, A.; Kim, E.; Song, C.; Murata, K.; Song, D.; Minagawa, J.; Liu, Z., Structural insight into light harvesting for photosystem II in green algae. Nat. Plants 2019, 5, 1320-1330. 